\title{
3D Finite Time Lyapunov Exponents in a left ventricle laboratory model
}

\author{
Maria Grazia Badas ${ }^{1, \mathrm{a}}$, Stefania Espa ${ }^{2}$, Stefania Fortini $^{2}$ and Giorgio Querzoli ${ }^{1}$ \\ ${ }^{1}$ DICAAR, University of Cagliari, 09123 Cagliari, Italy \\ ${ }^{2}$ DICEA, Sapienza Università di Roma, 00184 Roma, Italy
}

\begin{abstract}
Finite Time Lyapunov Exponents (FTLEs) are a powerful means to infer characteristic features of the flow that cannot be revealed by other Eulerian criteria. Recently FTLEs are becoming popular also in the medical context, for instance in the analysis of vascular flow measured by means of Magnetic Resonance Imaging. However, many of the FTLE experimental works are based only on two-dimensional velocity fields, moreover those computed on in-vivo data cannot be obtained under controlled and repeatable conditions. Here we present the 3D FTLE evolution inside a Left Ventricle (LV) laboratory model mimicking physiological human conditions. The investigation of FTLE fields highlights distinctive features of the cardiac flow and gives an insight on the physiological development of the Lagrangian Coherent Structures (LCS) that optimize the LV refill.
\end{abstract}

\section{Introduction}

Finite Time Lyapunov Exponents (FTLE, [1-2]) have been used in different context to characterize different regions of the flow and to identify the Lagrangian Choerent Structures (LCS). Moreover, recent contribution [3] highlighted how LCS patterns, apart from being strictly connected to the flow kinematics, are intimately linked to the identification of regions characterized by different energy fluxes. LCS are generally identified as FTLE field ridges, however [4] have shown how deriving LCS from FTLE fields requires strict mathematical conditions to be fulfilled. Although a one to one correspondence between FTLE and LCS cannot be considered, FTLE are a valuable means to give an insights on the evolution of a flow dynamics.

Their computation is well suited also for the analysis of experimental laboratory data, also due to its robustness with respect to measurement noise with respect to Eulerian criteria. Several experimental works dealing with FTLE are present in literature in different context spanning from atmospheric and oceanic flows, astrophysics, bio-fluid dynamics (see for instance [5-8]).

Recently, FTLE are becoming popular in medical context, for instance in in-vivo Magnetic Resonance and Doppler Echocardiography studies of the left ventricle [911]. In fact, it is well known that a proper formation and evolution of vortical structures inside the LV is a key factor helping the optimization of the blood circulation and ventricle wash out, whilst on the other hand, cardiac LV pathologies lead to abnormal LCS formation and evolution, causing presence of stagnation region or chaotic motion (see [12] and reference therein).

Hence in-vivo study aiming to identify, track and characterize vortical structure evolution and looking for the link among their features and physiological or pathological conditions are highly valuable.

However, there is the need for a term of reference for FTLE computation in the LV in controlled conditions helping the interpretation of results obtained in-vivo. In this perspective, investigation performed in laboratory models may represent an important means that may give possible future implication and feedback in diagnostic medical context.

Here, we present the evolution of 3D FTLE fields computed on a LV in-vitro model mimicking physiological conditions, where three dimensional velocity fields were measured by means of image analysis technique, as reported in [13]. Using the same experimental set-up, [14] already showed how important information can be inferred from 2D FTLE, however it is apparent how the comprehension of the complex asymmetric vortical structure evolving in the LV highly benefits from the availability of $3 \mathrm{D}$ velocity fields.

We here show how the conjunct analysis of the backward and forward 3D FTLE fields it is possible to delimit and track coherent structures evolving during the cardiac cycle, and how their evolution help for efficient mixing and recirculation. A comparison with another Eulerian criterion for vortical structures extraction, namely $\lambda_{2}$ computation, is also performed, showing how FTLE help to delimit the whole structure, instead of identifying only its core region.

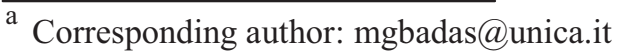




\section{Materials and methods}

\subsection{Experimental set-up}

We here briefly summarize the adopted experimental simulation and measurement procedure, however for an exhaustive description of the experimental set-up and velocity measurement technique, as well as an in-depth characterization of the flow emerging by means of Eulerian criteria the reader is referred to [13].

The sketch of the experimental set-up is shown in Figure 1. A flexible silicone rubber sack enclosed in a Plexiglas box mimics the behaviour of the left ventricle (LV). It is mounted on a on a rigid plate having two orifices that simulate the presence of mitral and aortic orifices, and are connected to a closed hydraulic circuit. Working fluid is water, and inside the LV it is seeded with neutrally buoyant particles to allow visualization. Thanks to the appropriate movement of a piston, electronically controlled, a physiological cycle inside the LV model is reproduced: the flow enters the mitral valve during diastolic phase determining the LV expansion and exits during the systolic phase. The model scale is $1: 1$ and the adimensional parameters, namely Reynolds and Womersly number, that govern the phenomenon are set to be within the physiological ranges.

A vertical laser sheet illuminates the measurement plane where images are taken by means of a high speed camera. Images were recorded on 24 several vertical planes in two orthogonal directions, Feature Tracking [15] was applied to track particles, in order to retrieve Lagrangian velocity fields, and then Eulerian ones on two-dimensional planes. Hence, the two-in plane velocity components fields were remapped on a regular threedimensional grid, phase average was then applied to data derived from 50 independent acquisition cycles in order to obtain velocity fields representative of a mean cardiac cycle.

Although the active role of ventricle tissue and leaflets cannot be simulated in such a model, previous works performed using the same experimental laboratory model [13-17] demonstrated how salient features of the LV hemodynamics observed in-vivo are satisfactory reproduced in this in-vivo model.

\subsection{Finite Time Lyapunov Exponents (FTLE) computation}

For an in-depth theoretical characterization of Finite Time Lyapunov Exponents (FTLE), the reader is referred to [1-2], while different techniques and schemes for optimizing their numerical computation, which can be highly demanding in terms of time and computational cost, and are revised in [18].

In this work FTLE computation was performed on experimental 3D velocity fields of the LV in-vitro model by means of a public domain code, NEWMAN [19].

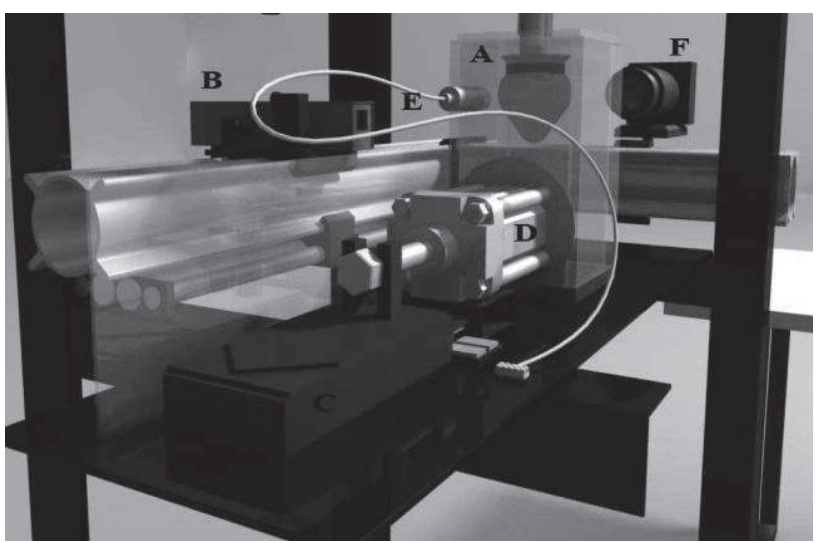

Fig. 1. Experimental set-up: ventricle chamber (A), laser (B), linear motor (C) driving the piston (D), pressure transducer (E), high speed camera $(\mathrm{F})$.

Specifically, for each time step of the cardiac cycle, a large number of synthetic particles is seeded and tracked, after integration of the Eulerian velocity fields, and the maximum linearized rate of exponential growth of adjacent particles during the prescribed integration time is computed. Namely, for each initial position $\mathbf{x}$ at a generic instant $t$ of the cycle, two fluid particles at initial distance $\delta \mathrm{r}$ are considered, and their maximum stretching $\|\delta \mathrm{r}\|_{\max }$ over time $\mathrm{T}$ is computed:

$$
\|\delta r\|_{\max }=e^{|T| \sigma_{T}(r, t)}\|\delta r\|
$$

where $\sigma_{\mathrm{T}}(\mathbf{x}, \mathrm{t})$ represents the FTLE value, i.e. the exponent of the exponential growth. For the adopted numerical scheme the reader is referred to [19].

Integration can be performed forward or backward in time: when computed forward in time FTLE fields help identifying repelling structures, whilst backward FTLE fields help to unveil attracting structures. Topological features emerging from FTLE fields are quite persistent with varying $\mathrm{T}$, even though ridges tend to become sharper with increasing integration time [2]. However, integration time has to be related to the characteristic flow time scales, hence we adopted $\mathrm{T}$ equal to the advection time scale, i.e. the ratio between the enddiastolic ventricle diameter and the peak mitral velocity. FTLE were computed on a spatial grid finer than the one of original velocity fields to better capture the FTLE ridges [9].

\section{Results}

Figure 2 shows the superposition of backward and forward 3D FTLE isosurfaces (respectively red and cyan surfaces) for some characteristic instants of the cardiac cycle. It is apparent how this representation helps the visualization of the coherent vortex structures and their evolution. At the beginning of the first wave of the diastolic phase of the cardiac cycle, called E-wave, a vortex ring is originated due to the jet entering the mitral orifice (Figure 2a-b). 

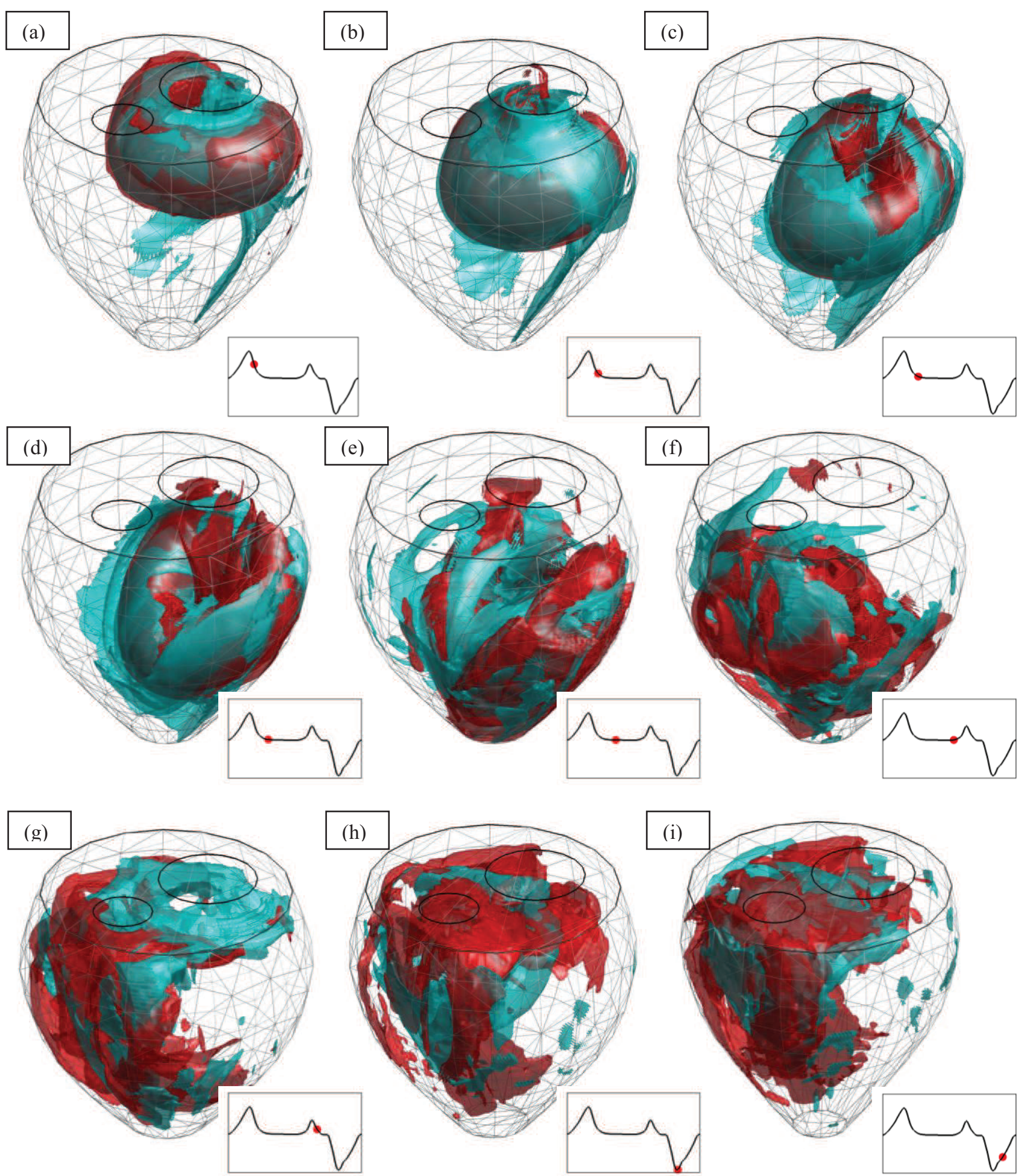

Fig. 2. Superposition of isovalues of backward (cyan) and forward (red) FTLE fields at different instants of the cardiac cycle, grey lines identify the LV, the bigger circle is the mitral orifice, while the smaller one is the aortic orifice. The small panel next to each plot shows the considered instant (red dot) of the cycle. Plots a-b refer to the first diastolic jet (E-wave), plots c-d show the progressive distortion of the vortex ring and its impingement on the ventricle wall, plots e-f the structure rupture during the diastasis and its redirection towards the aortic orifice, plot g shows the second vortex ring generating at the mitral orifice and corresponding to A-wave, plots h-i show the systolic phase when a single structure is being ejected from the aortic orifice.

Apart from some spurious effects, the superposition of the two isosurfaces structure appears to enclose a well defined region of the flow, moreover there is a patch of the forward FTLE which exits this confined region and is pushed out towards the ventricle wall, this corresponds to the point of impingement of the vortical structure on the LV boundary.

At a following instant of the cycle (Figure 2c) the region enclosing the vortex ring, which before appeared symmetrical, is deformed forming and inclined and 
asymmetrical structure, which then collides against the ventricle wall (Figure 2d) as already predicted from past FTLE fields. Afterwards (Figure 2e-f) the well organized structure brakes and its remaining parts start rotating in a clockwise sense toward the aortic orifice. When the second diastolic jet, called A-wave, enters the mitral orifice a new vortex ring forms and is well identified from the superposition of forward and backward fields (Figure 2g). This new structure follows the track of the previous one, and helps to push it towards the aortic orifice, where a single vortical structure extends from the apex up to the aortic orifice in left part of the LV is directed and ejected during the systolic phase (Figure gh). However, in this last remaining part of the cycle the FTLE fields appear to be much less clear, this is due to the E-wave vortex ring rupture and to the systolic ejection from the aortic orifice.

The backward FTLE fields act as attracting barriers for the flow, as an example, Figure 3 portrays for two instant of the cycle, a vertical diametric section of the backward FTLE superimposed to synthetic particles injected at the mitral orifice during the E-wave (green points) and A-wave (yellow points). These plots confirm how the particles well follow the FTLE surfaces, that are hence useful to identify and distinguish different dynamic regions of the flow.

In Figure 4 we compare FTLE to another Eulerian criteria used to identify vortical structure, namely $\lambda_{2}$ computation: the plots, obtained for three instants during the E-wave, show $\lambda_{2}$ isovalues superimposed to the backward (left column, panels a, c, e) or forward 3D FTLE (right column, panels b, d, f) sectioned with a diametric vertical plane. All the panels show how the two FTLE surfaces enclose $\lambda_{2}$ region, helping to delimit and track the whole vortical region instead of its core. In the left panels we see how the backward FTLE field form a virtual channel connected to the mitral orifice that redirects the E-wave particles towards the LV wall, and how the structure is deformed and propagates asymmetrically. In addition, forward FTLE fields in Figure $4 \mathrm{~d}-\mathrm{f}$, highlight, better than in Figure 2, the point of impingement of the vortex ring onto the ventricle wall.

We recall here that the right part of the structure appears to be cut because the experimental data do not cover the region adjacent to the ventricle wall.

\section{Conclusions}

Experimental three-dimensional velocity data measured in a laboratory model mimicking physiological LV conditions were analyzed to investigate FTLE fields evolution in controlled conditions. Even though caution has to be taken when inferring LCS from FTLE data, their analysis is an important aid for capturing vortical structures and complements the information given by other Eulerian criteria.

The conjunct analysis of backward and forward 3D FTLE fields gives a full picture of the asymmetrical LCS development, that initially follows the mitral orifice one, then progressively stretches and moves following a diagonal propagation direction. Backward FTLE ridges create a virtual channel connected to the mitral orifice, that drives blood flow, while the structure folds onto the ventricle wall creating the recirculation path that facilitates subsequent ejection. Although important information can be inferred from 2D FTLE as previously shown in [14], it is apparent how the comprehension of the complex asymmetric vortical structure highly benefits from the availability of $3 \mathrm{D}$ velocity fields.

Our results are in agreement with the phenomenological pictured emerging in both clinical and laboratory studies, and in particular in MRI ones. Thus FTLE analysis in a LV laboratory model appears to be a promising way to investigate in controlled and repeatable conditions the mutual relationships between the LCS formation and evolution and the LV pathophysiological conditions.

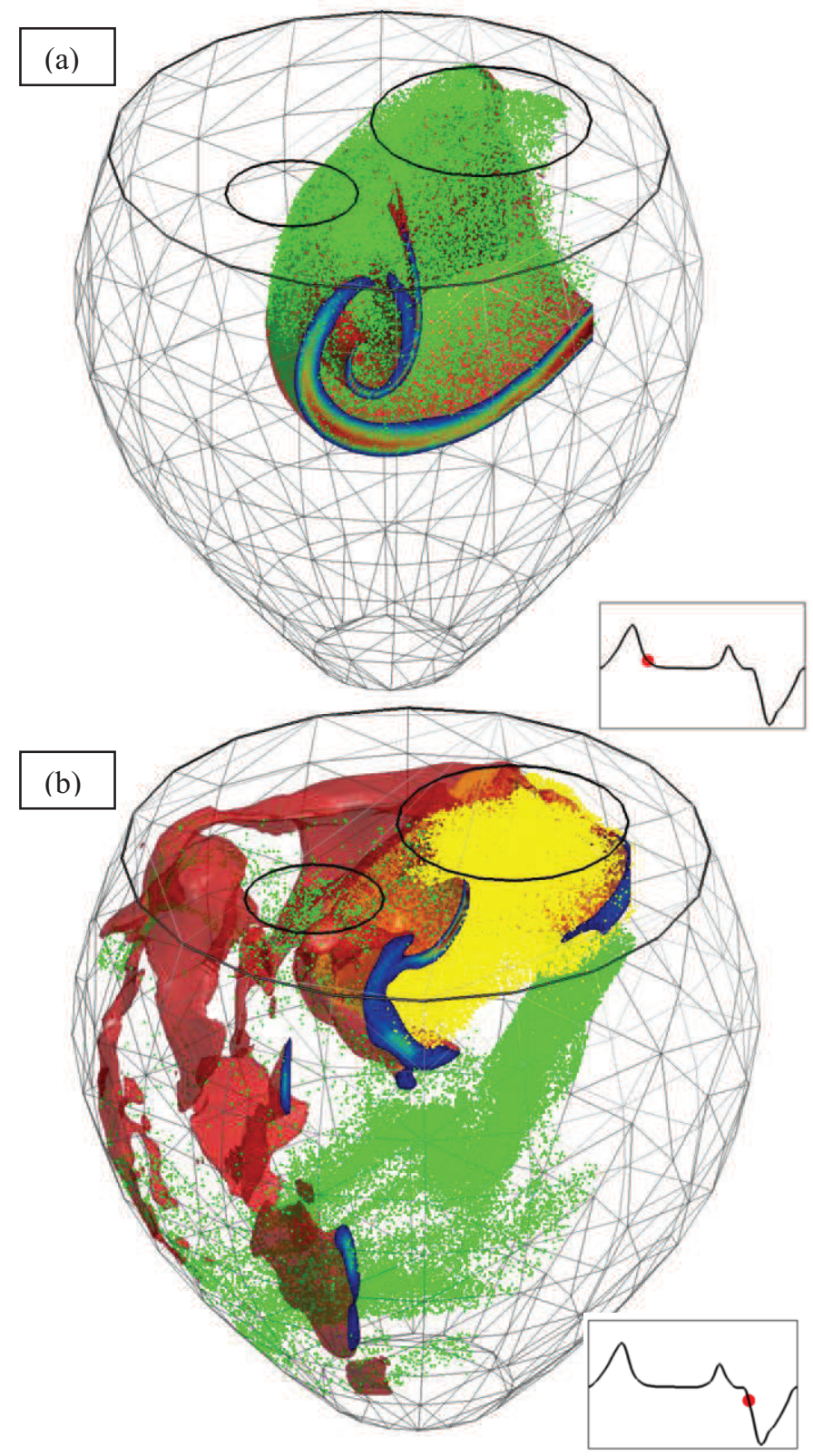

Fig. 3. Diametric 3D section of backward FTLE isovalue field is superimposed to the particle position for two instants of the cycle, at the end of the E-wave (plot a) and at the beginning of systole. Particles were injected during the E-wave (green dots) and A-wave (yellow dots). 
(a)
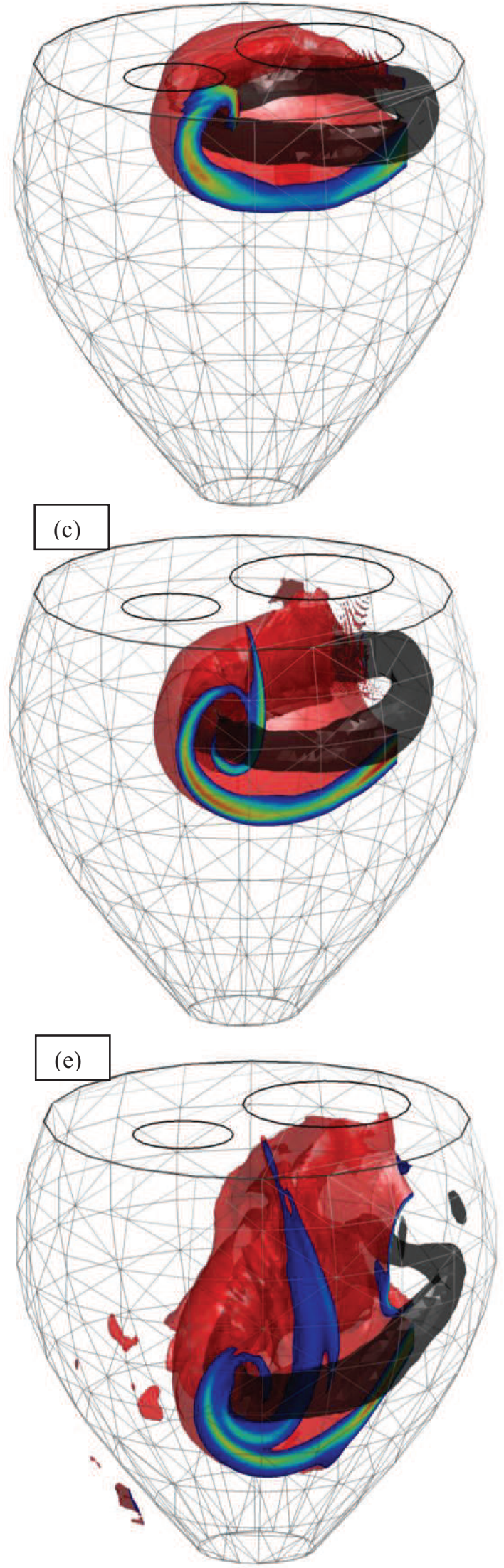

(b)

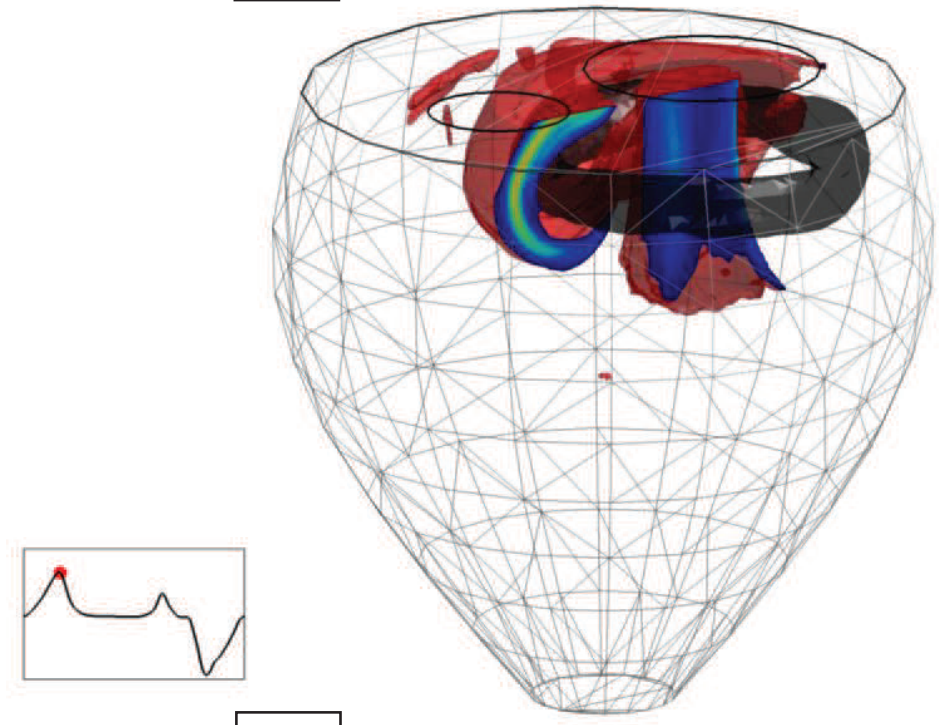

(d)

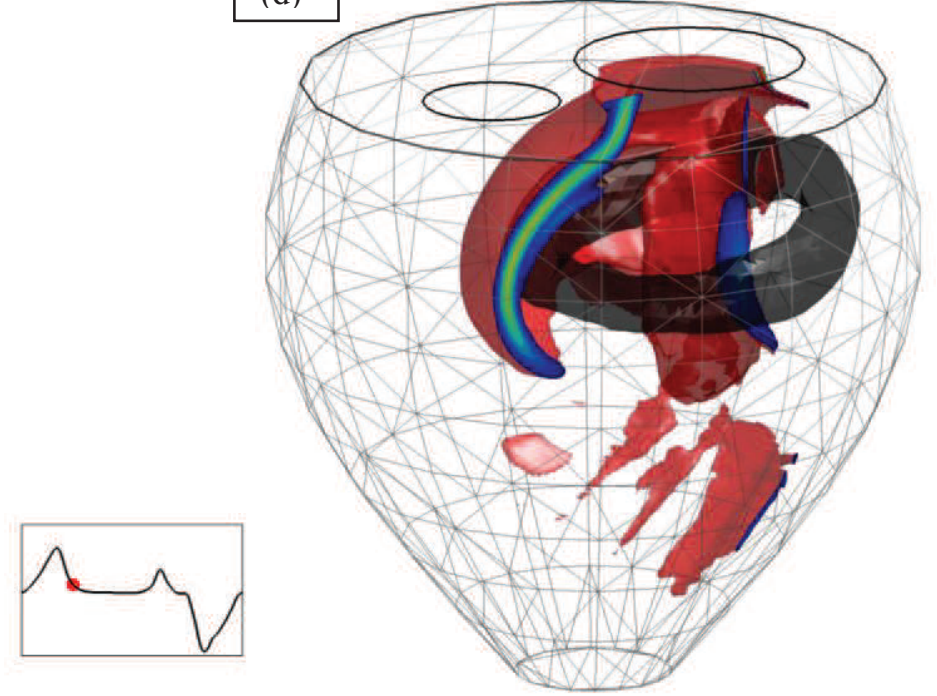

(f)
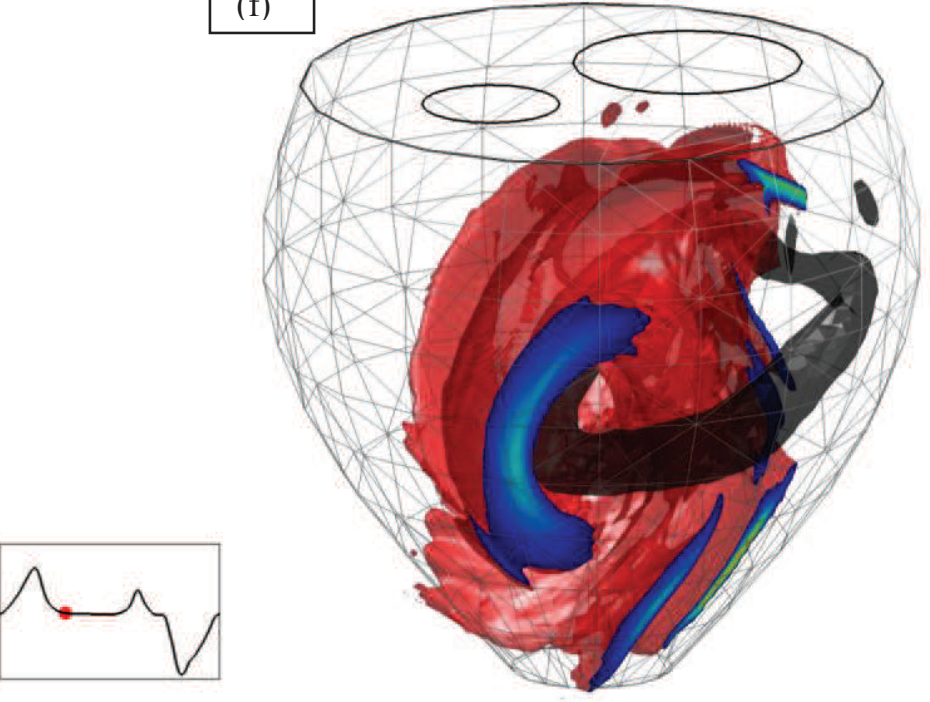

Fig. 4. Diametric 3D section of backward (left column - plots a,c,e) or forward (right column - plots b,d,f) FTLE isovalue fields (red rurfaces) for three instants during the E-wave (as indicated from the red dot in the small inset) are superimposed to the $\lambda_{2}$ isovalue field (grey surface). 


\section{References}

1. G. Haller, Phys. D. 149 (2001).

2. S.C. Shadden, F. Lekien, J. E. Marsden. Phys. D. 212 (2005).

3. D. H. Kelley, M. R. Allshouse, N. T. Ouellette, Phys Rev E, 88 (2013)

4. G. Haller, Phys. D. 240 (2011).

5. M. G. Badas, G. Querzoli, Exp Fluids 50(4) (2011).

6. F. J. Beron Vera, Y. Wang, M. J. Olascoaga, G. J. Haller, J. Phys. Oceanogr. 43(7) (2013)

7. J. Vétel, A. Garon, D. Pelletier, Exp Fluids 46(6) (2009)

8. P. Miron, J. Vétel, A. Garon, Exp Fluids 55 (2014)

9. S. Hendabadi, J. Bermejo, Y. Benito, R. Yotti, F. Fernandez-Avilés, J. C. del Alamo, S. Shadden, Ann. Biomed. Eng., 41 (12) (2013)

10. Töger, J., M. Carlsson, G. Soderlind, H. Arheden, and E. Heiberg. BMC Medical Imaging. 11:10 (2011).

11. J. J. Charonko, R. Kumar, K. Stewart, W. C. Little, P. P. Vlachos, Ann. Biomed. Eng., 41 (5) (2013)

12. A. Kheradvar, G. Pedrizzetti, Vortex formation in the cardiovascular system, Springer (2012)

13. S. Fortini, G. Querzoli, S. Espa, A. Cenedese, Exp Fluids 54(1) (2013)

14. S. Espa, M. G. Badas, S. Fortini, G. Querzoli, A. Cenedese EJM B/Fluids, 35 (2012)

15. A. Cenedese, Z. Del Prete, M. Miozzi, G. Querzoli, Exp Fluids, 39 (2005).

16. G. Querzoli, A. Cenedese, S. Fortini, Phys. Fluids 22 (2010)

17. S. Espa, S. Fortini, G. Querzoli, A. Cenedese, J. Vis, 16(4) (2013)

18. F. Sadlo, A. Rigazzi, R. Peikert, Time-dependent visualization of lagrangian coherent structures by grid advection, Topological Methods in Data Analysis and Visualization (Springer Berlin Heidelberg, 2011)

19. Du Toit, P. C. and J. E. Marsden, Journal of Fixed Point Theory and Applications, 7(2) (2010). 